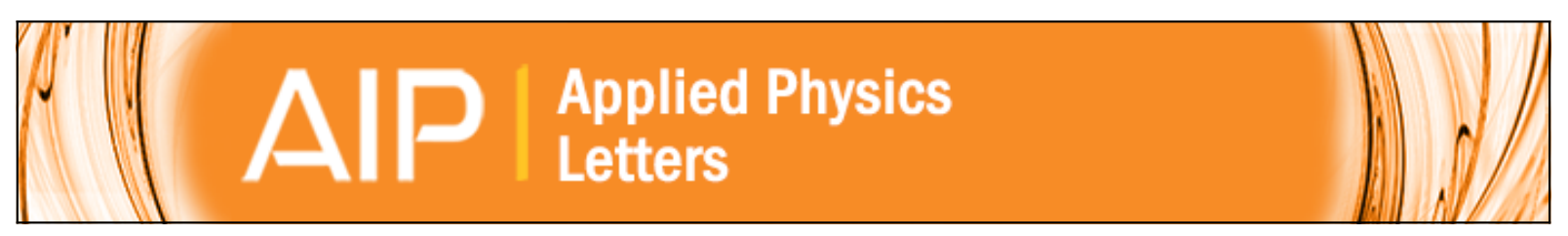

\title{
Magnetocapacitance of an electrically tunable silicene device
}

M. Tahir and U. Schwingenschlögl

Citation: Applied Physics Letters 101, 132412 (2012); doi: 10.1063/1.4754711

View online: http://dx.doi.org/10.1063/1.4754711

View Table of Contents: http://scitation.aip.org/content/aip/journal/apl/101/13?ver=pdfcov

Published by the AIP Publishing

MULTIPHYSICS

SIMULATION

S̈PECTRUM

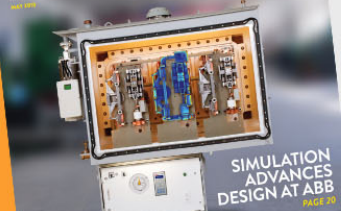

FREE Multiphysics Simulation e-Magazine
DOWNLOAD TODAY 》 


\title{
Magnetocapacitance of an electrically tunable silicene device
}

\author{
M. Tahir and U. Schwingenschlögl $\left.\right|^{\mathrm{a})}$ \\ PSE Division, KAUST, Thuwal 23955-6900, Kingdom of Saudi Arabia
}

(Received 27 July 2012; accepted 11 September 2012; published online 26 September 2012)

\begin{abstract}
Despite their structural similarity, the electronic properties of silicene are fundamentally different from those of well-known graphene due to the strong intrinsic spin orbit interaction and buckled structure of silicene. We address the magnetocapacitance of spin and valley polarized silicene in an external perpendicular magnetic field to clarify the interplay of the spin orbit interaction and the perpendicular electric field. We find that the band gap is electrically tunable and show that the magnetocapacitance exhibits beating at low and splitting of the Shubnikov de Haas oscillations at high magnetic field. (C) 2012 American Institute of Physics. [http://dx.doi.org/10.1063/1.4754711]
\end{abstract}

Silicene forms an alternative platform to explore the properties of two-dimensional electronic systems. ${ }^{1-9}$ Its honeycomb lattice is isostructural to graphene with a low energy Dirac spectrum. Indeed, silicene exhibits advantages over graphene in several areas of intense recent research due to the strong spin orbit interaction (SOI) and its buckled structure. The latter is related to the large ionic radius of silicon. Although lots of efforts had been undertaken to induce in graphene a band gap ${ }^{10,11}$ and substantial SOI,${ }^{12-14}$ no satisfactory progress was achieved yet. ${ }^{15,16}$ Overcoming these problems, silicene exhibits a large band gap of $1.55 \mathrm{meV}$, which provides a mass to the Dirac fermions. ${ }^{17-19}$ In addition, the band gap can be increased by the application of a perpendicular electric field along the buckling direction. ${ }^{20}$ Strong SOI combined with a tunable band gap are expected to be key criteria in the race towards electronic applications compatible with the existing technology.

There are two major distinctions between silicene and graphene: First, the inversion symmetry is broken due to the electric field in the direction of the buckling, see Fig. 1. This field give rise to the valley Hall effect, ${ }^{21}$ where particles in different valleys flow to opposite transverse edges. Second, the strong SOI results in a quantum spin Hall effect. ${ }^{17}$ In the presence of a perpendicular external magnetic field, a valley polarized quantum Hall effect has been demonstrated recently. ${ }^{22}$ In this context, capacitance measurements are among the most important tools for studying the electronic properties. They can be effectively used to probe the thermodynamic density of states (DOS). Although the focus in silicene research has been on transport properties, insight into the fundamental electronic properties and device physics calls for knowledge about the capacitance-voltage (C-V) characteristics of the system. Results have been reported for carbon nanotubes, graphene nanoribbons, and mono- and bilayer graphene systems. ${ }^{23-28} \mathrm{In}$ view of this, attention is now being paid to electrostatic properties, such as the magnetocapacitance of silicene devices. Furthermore, to improve the performance of field effect transistors, ${ }^{29}$ silicene is creating excitement as potential channel material. This is due to the excellent intrinsic transport features as well as the possibility of patterning device structures by top-down lithographical techniques.

\footnotetext{
${ }^{\text {a) }}$ Author to whom correspondence should be addressed. Electronic mail: udo.schwingenschlogl@kaust.edu.sa. Tel.: +966(0)544700080.
}

The present work aims at determining the combined effects of the SOI and perpendicular electric field on the magnetocapacitance of a silicene device. To this aim, we model silicene by an effective Hamiltonian in the xy-plane with an external magnetic field $B$ in z-direction, taking into account the effects of the SOI and perpendicular electric field. The Dirac fermions in buckled silicene obey the twodimensional graphene-like Hamiltonian ${ }^{18,20-22}$

$$
H_{s}^{\eta}=v\left(\eta \sigma_{x} \mathbf{p}_{x}+\sigma_{y} \mathbf{p}_{y}\right)+\eta s \lambda \sigma_{z}+\Delta \sigma_{z}
$$

with $\eta=+1 /-1$ for $K / K^{\prime}, s=+1 /-1$ for spin up/down, the Pauli matrices $\sigma_{x}, \sigma_{y}$, and $\sigma_{z}$, the Fermi velocity $v$ of the Dirac fermions, and the two dimensional canonical momentum p. The uniform electric field $E_{z}$ perpendicular to the silicene sheet $(l=0.23 \AA)$ results in the energy $\Delta=2 l E_{z}$. The intrinsic spin orbit coupling strength is $\lambda=4 \mathrm{meV} .^{18,19}$ Using the Landau gauge with vector potential $(0, B x, 0)$, the diagonalization of the Hamiltonian given in Eq. (1) leads to eigenvalues

$$
\begin{aligned}
& E_{s, 0}^{\eta}=\eta \Delta+s \lambda \text { for } n=0 \\
& E_{s, n, t}^{\eta}=t \sqrt{n \hbar^{2} \omega^{2}+(\Delta+\eta s \lambda)^{2}} \text { for } n \neq 0 .
\end{aligned}
$$

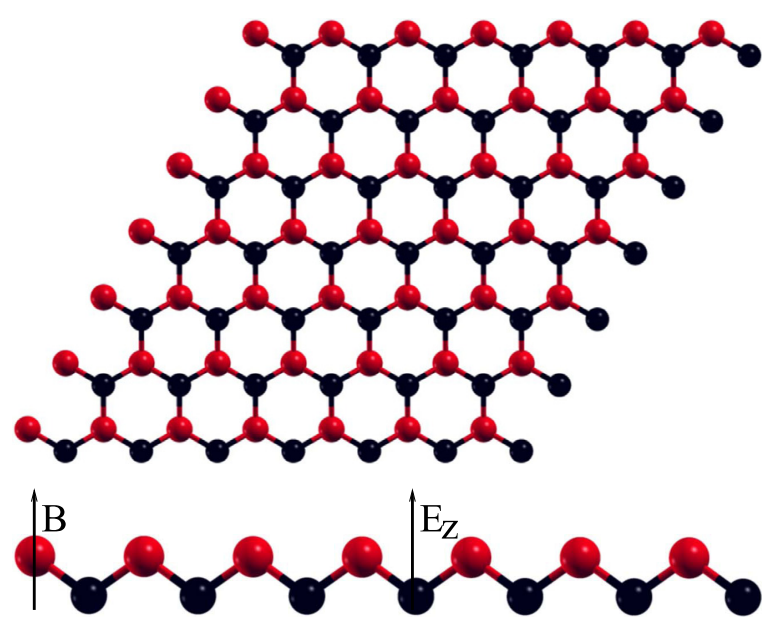

FIG. 1. Crystal structure of silicene. The black and red spheres represent the two sublattices. The directions of the electric and magnetic fields are indicated by arrows. 
Here, $t=+1 /-1$ represents the electron/hole band, $\omega=v \sqrt{2 e B / \hbar}$, and $n$ is the Landau level index. We note that a splitting of the Landau levels requires finite SOI and finite electric field. In this case, the $n=0$ Landau level explicitly lifts the four-fold degeneracy known from graphene. In the absence of an electric field, the spectrum is electron-hole symmetric with a SOI gap. Finally, the eigenvalues depend rather linearly on $B$ (and are not proportional to $\sqrt{B}$ ) as the Dirac fermions acquire mass due to a relatively large SOI gap. This mass can be controlled by application of an external perpendicular electric field.

We consider a gated silicene device in which the capacitor is formed between the gate and the silicene. The magnetocapacitance $C_{Q}{ }^{27,30}$ is the dominant capacitive contribution in nanoscale devices and represents the charge response in the channel as the channel potential is varied. In the limit of zero temperature, it is obtained analogous to Ref. 27 as

$$
\begin{aligned}
C_{Q}= & \frac{e^{2}}{2 \pi l^{2}}\left\{\sum_{\eta, s} \frac{1}{\Gamma \sqrt{2 \pi}} \exp \left[-\frac{\left(E_{F}-E_{s, 0}^{\eta}\right)^{2}}{2 \Gamma^{2}}\right]\right. \\
& \left.+\sum_{\eta, s, t} \sum_{n=1}^{\infty} \frac{1}{\Gamma \sqrt{2 \pi}} \exp \left[-\frac{\left(E_{F}-E_{s, n, t}^{\eta}\right)^{2}}{2 \Gamma^{2}}\right]\right\},
\end{aligned}
$$

where $\Gamma$ is the width of the Gaussian broadening of the Landau levels. The magnetocapacitance is plotted in Fig. 2 as a function of the Fermi energy (i.e., of the gate voltage) for $B=1 \mathrm{~T}, \lambda=4 \mathrm{meV}$, and $\Delta=8 \mathrm{meV}$. We choose a constant broadening of $\Gamma=0.5 \mathrm{meV}$. We are interested in changes of the character at $E_{F}=0$ on variation of the SOI and electric field energy relative to each other. It must be noted that the $n=0$ Landau level here plays the most important role. Figs. 2 and 3 show that $C_{Q}$ is zero at $E_{F}=0$. This occurs because the $n=0$ Landau level splits only when $\lambda \neq 0$ or $\Delta \neq 0$. It splits into one electron and one hole level in each valley independently (individual electron-hole symmetry) when $\lambda=0$ and $\Delta \neq 0$. Importantly, $C_{Q}$ can be tuned in each valley independently from a minimum to a maximum when the electric field energy is enhanced via the external gate

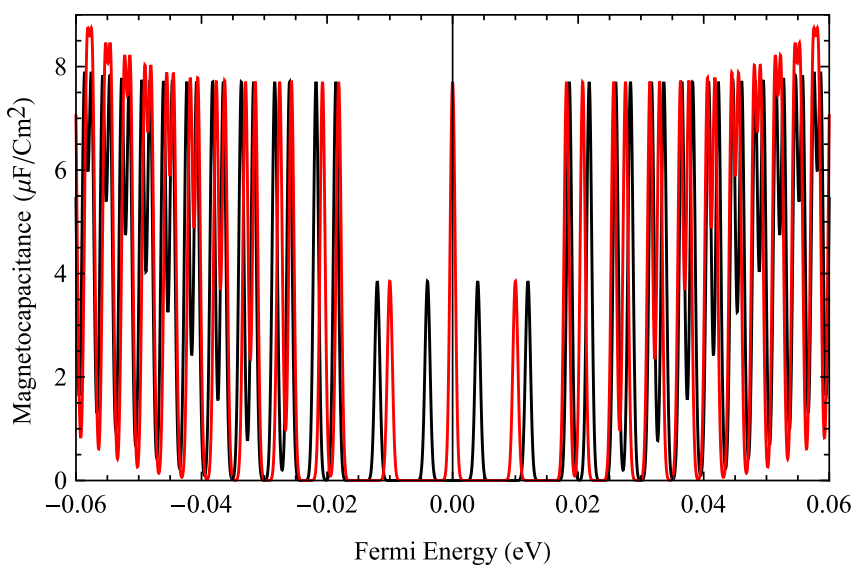

FIG. 3. Total magnetocapacitance of the $\mathrm{K}$ and $\mathrm{K}^{\prime}$ valleys as a function of the Fermi energy for $B=1 \mathrm{~T}$, SOI energy $\lambda=4 \mathrm{meV}$, and electric field energy $\Delta=8 \mathrm{meV}$ (black) and $\Delta=4 \mathrm{meV}$ (red).

voltage. For $\Delta>\lambda$, both $n=0$ sublevels are shifted into the electron/hole region for the $\mathrm{K} / \mathrm{K}^{\prime}$ valley, see Fig. 2. To observe the splitting, the broadening of the Landau levels must be less than the electric field energy. We note that not only the $n=0$ Landau level but also all Landau levels are split into two sublevels. The total magnetocapacitance obtained by summing over both valleys is shown in Fig. 3 as a function of the Fermi energy for $\Delta>\lambda$ and $\Delta=\lambda$. An electrically tunable band gap at $E_{F}=0$ is evident. The magnetocapacitance is zero for the former case and maximal (with a single peak and electron-hole symmetric spectrum) for the latter case. All other Landau levels are likewise split. In the case of maximal magnetocapacitance, the energy gap between the spin down states closes to yield a sharp peak at $E_{F}=0$, whereas the spin up states maintain their energy gap. This behavior is consistent with Eq. (2).

In Fig. 4, we show the Shubnikov de Haas oscillations in $C_{Q}$ for $T=0 \mathrm{~K}, \lambda=4 \mathrm{meV}, \Gamma=0.5 \mathrm{meV}$, and realistic values of $n_{e}=5 \times 10^{15} \mathrm{~m}^{-2}$ and $v=5 \times 10^{5} \mathrm{~ms}^{-1} \cdot{ }^{17-19}$ For low and high magnetic field, we observe a beating pattern and a splitting of the Shubnikov de Haas oscillations, respectively. Both is the result of interference of the frequencies of the spin up and spin down states and therefore a consequence

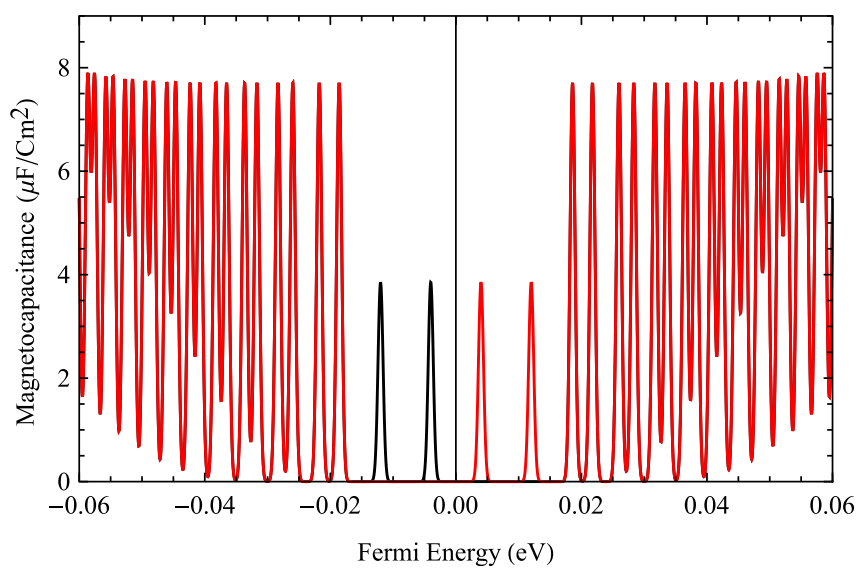

FIG. 2. Magnetocapacitance as a function of the Fermi energy for $B=1 \mathrm{~T}$, SOI energy $\lambda=4 \mathrm{meV}$, and electric field energy $\Delta=8 \mathrm{meV}$. Red/black color represents the $\mathrm{K} / \mathrm{K}^{\prime}$ valley.

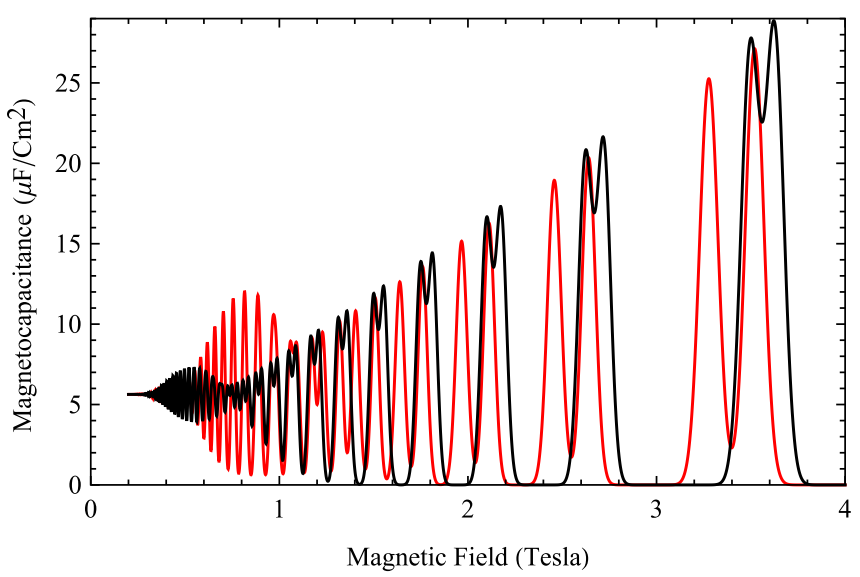

FIG. 4. Total magnetocapacitance of the $\mathrm{K}$ and $\mathrm{K}^{\prime}$ valleys as a function of the magentic field for SOI energy $\lambda=4 \mathrm{meV}$ and electric field energy $\Delta=$ $8 \mathrm{meV}$ (black) and $\Delta=15 \mathrm{meV}$ (red). 
of the splitting of the Landau levels for finite SOI and finite electric field. The beating pattern vanishes once the electric field energy dominates the SOI energy. For the chosen parameters, this occurs at a magnetic field of about $1.5 \mathrm{~T}$. Above this value, we find a well resolved splitting of the Shubnikov de Haas oscillations in Fig. 4, which we next investigate in some more detail. With the help of the Poisson summation formula, we obtain for the contributions of the $n \geq 1$ Landau levels for the oscillatory part in the magnetocapacitance

$$
C_{Q} \sim 4 \cos \left[\frac{2 \pi}{\hbar^{2} \omega^{2}}\left(E_{F}^{2}-\lambda^{2}-\Delta^{2}\right)\right] \cos \left[\frac{4 \pi}{\hbar^{2} \omega^{2}} \lambda \Delta\right] .
$$

This result represents a wave of higher frequency with an amplitude oscillating at lower frequency. Both the observed beating pattern and splitting of Shubnikov de Haas oscillations are induced, because $\lambda \Delta \ll E_{F}$. The amplitude is modulated by $\cos \left[\frac{4 \pi}{\hbar^{2} \omega^{2}} \lambda \Delta\right]$ and nodes occur at $\frac{4}{\hbar^{2} \omega^{2}} \lambda \Delta$ $= \pm 0.5, \pm 1.5, \ldots$, where the modulating cosine vanishes as shown in Fig. 4. We also note that the amplitude modulation given by the term $\cos \left[\frac{4 \pi}{\hbar^{2} \omega^{2}} \lambda \Delta\right]$ occurs only when both the SOI and electric field are finite. Moreover, the threshold magnetic field where beating is seen depends on both $\lambda$ and $\Delta$. For magnetic fields up to about $1.5 \mathrm{~T}$, the beating persists. Above this value, it is quenched to show splitting of the Shubnikov de Haas oscillations.

In conclusion, we have investigated the effects of SOI and electric field on the magnetocapacitance of silicene, a monolayer of silicon, in a magnetic field. It turns out that the SOI induces a large band gap at the charge neutrality point and that the electric field breaks the inversion symmetry. In addition, we have shown that the presence of both SOI and electric field yields for the magnetocapacitance a beating pattern at low and a level splitting at high magnetic fields. This behavior is explained by interference of the Shubnikov de Haas oscillations at the two frequencies of the split Landau levels. Noting that the cyclotron energy for $B=1 \mathrm{~T}$ is $\hbar \omega=18 \mathrm{meV}$, observation of the Landau level splitting and the discussed consequences requires that the temperature and disorder broadening do not become comparable to the SOI and electric field energies. We note that our analysis for silicene is also valid for germanene, which is a monolayer of germanium with an even stronger SOI of $\lambda=43 \mathrm{meV} .{ }^{17-19}$
${ }^{1}$ K. Takeda and K. Shiraishi, Phys. Rev. B 50, 14916 (1994).

${ }^{2}$ G. G. Guzmán-Verri and L. C. Lew Yan Voon, Phys. Rev. B 76, 075131 (2007).

${ }^{3}$ S. Cahangirov, M. Topsakal, E. Aktürk, H. Şahin, and S. Ciraci, Phys. Rev. Lett. 102, 236804 (2009).

${ }^{4}$ H. Şahin, S. Cahangirov, M. Topsakal, E. Bekaroglu, E. Aktürk, R. T. Senger, and S. Ciraci, Phys. Rev. B 80, 155453 (2009).

${ }^{5}$ B. Aufray, A. Kara, S. Vizzini, H. Oughaddou, C. Léandri, B. Ealet, and G. L. Lay, Appl. Phys. Lett. 96, 183102 (2010).

${ }^{6}$ P. E. Padova, C. Quaresima, C. Ottaviani, P. M. Sheverdyaeva, P. Moras, C. Carbone, D. Topwal, B. Olivieri, A. Kara, H. Oughaddou, B. Aufray, and G. L. Lay, Appl. Phys. Lett. 96, 261905 (2010).

${ }^{7}$ B. Lalmi, H. Oughaddou, H. Enriquez, A. Kara, S. Vizzini, B. Ealet, and B. Aufray, Appl. Phys. Lett. 97, 223109 (2010).

${ }^{8}$ P. Vogt, P. D. Padova, C. Quaresima, J. Avila, E. Frantzeskakis, M. C. Asensio, A. Resta, B. Ealet, and G. L. Lay, Phys. Rev. Lett. 108, 155501 (2012).

${ }^{9}$ A. Fleurence, R. Friedlein, T. Ozaki, H. Kawai, Y. Wang, and Y. Y. Takamura, Phys. Rev. Lett. 108, 245501 (2012).

${ }^{10}$ A. Bostwick, F. Speck, T. Seyller, K. Horn, M. Polini, R. Asgari, A. H. MacDonald, and E. Rotenberg, Science 328, 999 (2010).

${ }^{11}$ D. C. Elias, R. V. Gorbachev, A. S. Mayorov, S. V. Morozov, A. A. Zhukov, P. Blake, L. A. Ponomarenko, I. V. Grigorieva, K. S. Novoselov, F. Guinea, and A. K. Geim, Nat. Phys. 7, 701 (2011).

${ }^{12}$ C. L. Kane and E. J. Mele, Phys. Rev. Lett. 95, 226801 (2005).

${ }^{13}$ H. Min, J. E. Hill, N. A. Sinitsyn, B. R. Sahu, L. Kleinman, and A. H. MacDonald, Phys. Rev. B 74, 165310 (2006).

${ }^{14}$ Y. Yao, F. Ye, X. Qi, S. Zhang, and Z. Fang, Phys. Rev. B 75, 041401(R) (2007).

${ }^{15}$ A. Varykhalov, J. S. Barriga, A. M. Shikin, C. Biswas, E. Vescovo, A. Rybkin, D. Marchenko, and O. Rader, Phys. Rev. Lett. 101, 157601 (2008).

${ }^{16}$ C. Weeks, J. Hu, J. Alicea, M. Franz, and R. Q. Wu, Phys. Rev. X 1, 021001 (2011).

${ }^{17}$ C. C. Liu, W. Feng, and Y. Yao, Phys. Rev. Lett. 107, 076802 (2011).

${ }^{18}$ C. C. Liu, H. Jiang, and Y. Yao, Phys. Rev. B 84, 195430 (2011).

${ }^{19}$ N. D. Drummond, V. Zólyomi, and V. I. Fal'ko, Phys. Rev. B 85, 075423 (2012).

${ }^{20}$ M. Ezawa, New J. Phys. 14, 033003 (2012).

${ }^{21}$ M. Tahir, A. Manchon, K. Sabeeh, and U. Schwingenschlögl, e-print arXiv:1206.3650v1 (2012).

${ }^{22} \mathrm{M}$. Tahir and U. Schwingenschlögl, e-print arXiv:1207.4745v1 (2012).

${ }^{23}$ S. Ilani, L. A. K. Donev, M. Kindermann, and P. L. McEuen, Nat. Phys. 2, 687 (2006).

${ }^{24}$ J. Guo, Y. Yoon, and Y. Ouyang, Nano Lett. 7, 1935 (2007).

${ }^{25}$ J. Xia, F. Chen, J. Li, and N. Tao, Nat. Nanotechnol. 4, 505 (2009).

${ }^{26}$ F. Giannazzo, S. Sonde, V. Raineri, and E. Rimini, Nano Lett. 9, 23 (2009).

${ }^{27}$ L. A. Ponomarenko, R. Yang, R. V. Gorbachev, P. Blake, A. S. Mayorov, K. S. Novoselov, M. I. Katsnelson, and A. K. Geim, Phys. Rev. Lett. 105, 136801 (2010).

${ }^{28}$ H. Xu, Z. Zhang, and L. M. Peng, Appl. Phys. Lett. 98, 133122 (2011).

${ }^{29}$ T. Fang, A. Konar, H. L. Xing, and D. Jena, Appl. Phys. Lett. 91, 092109 (2007).

${ }^{30}$ M. Tahir and U. Schwingenschlögl, Appl. Phys. Lett. 101, 013114 (2012). 